3. For overviews of this work, see: Bernard, E., Cameron, S. 2016. Advancing HIV Justice 2: Building momentum in global advocacy against HIV criminalisation. HIV Justice Network and GNP+. Brighton/Amsterdam, http://www.hivjustice.net/wp-content/ uploads/2016/05/AHJ2.final2_.10May2016.pdf; Mykhalovskiy, E. (2015). The public health implications of HIV criminalization: past, current, and future research directions. Critical Public Health, 25:4, 373-385; Harason, D. Galletly, C., O'Keefe, E., Lazzarini, Z. 2017. Criminalization of HIV Exposure: A Review of Empirical Studies in the United States, AIDS and Behaviour, $21: 27,27-50$
4. Jasanoff S. Just evidence: the limits of science in the legal process. J Law Med Ethics 2006; 34:328-341.

5. See, among many: Alexander, M. (2012). The New Jim Crow: Mass Incarceration in Age of Colorblindness. The New Press: New York; Simon, J. (2007). Governing Through Crime: How the War on Crime Transformed American Democracy and Created a Culture of Fear. Oxford University Press: London; Razack, S. (2015). Dying from improvement: Inquests and inquiries into Indigenous deaths in custody. Toronto: University of Toronto Press.

DOI:10.1097/QAD.0000000000001570

\title{
Dolutegravir-based regimen maintains virological success in a patient with archived mutations to integrase inhibitors
}

Dolutegravir (DTG) is the latest integrase inhibitor (INI) to be approved for the treatment of HIV-1 infection, with a high antiviral effect, excellent efficacy and safety profile $[1,2]$. Although DTG seems to show a high genetic barrier, its resistance profile has not yet been established, particularly in patients with failure on an INI-based regimen [3]. The VIKING-3 study among patients with INI-resistant virus reported that the maximum reduction in DTG susceptibility occurred when Q148 was accompanied by at least two other major mutations. For these patients, the odds of achieving HIV-RNA less than 50 copies $/ \mathrm{ml}$ were $96 \%$ lower than those of patients with no evidence of Q148 mutations, even when DTG was dosed twice a day (BID) [4,5]. Moreover, the role of archived integrase mutations is still unknown and generally the clinical significance of drug resistance mutations (DRMs) in proviral DNA remains controversial. Several studies showed that DRMs in proviral DNA may be implicated in therapy failure, whereas others reported that genotypic resistance tests on peripheral blood mononuclear cells (PBMCs) did not provide useful information for assessing the risk of virological failure [6-8].

Here, we describe the case of a successful treatment obtained with a DTG-containing regimen in a 55-yearold MSM, harboring mutations archived in PBMCs conferring high-level resistance to the newer INI in the presence of a multidrug class-resistant HIV-1 because of more than 20 years' antiretroviral therapy (ART).

The man has been living with HIV-1 (subtype B) since 1986, with a history of AIDS-defining events and prior failure to four antiretroviral classes [nucleos(t)ide and nonnucleoside reverse transcriptase inhibitors, protease inhibitors, and fusion inhibitors]. He started a raltegravir (RAL)-containing regimen in June 2013, but did not take it on regular basis and failed to achieve a fully suppressed viral load. One year later, resistance to INIs was detected in plasma (E138A, G140S, Q148H); RAL was stopped and since then his treatment has been modified several times according to drug resistance and intolerance. Because of persistent viremia, in February 2015, genotypic resistance typing (GRT) was performed on plasma and PBMCs, revealing the same mutations in both integrase (IN) gene compartments. On the basis of GRT, a therapeutic regimen containing rilpivirine and abacavir/lamivudine was started. Viral load (VL) decreased slightly thereafter, and in December 2015, another GRT performed on plasma disclosed no major DRM in the IN gene. Despite a previous INI failure, due to few therapeutic options, the patient received DTG (BID), emtricitabine and tenofovir disoproxil fumarate. A rapid virological response was observed, and after 3 months, HIV-RNA declined below 37 copies $/ \mathrm{ml}$. Viremia was monitored every 12 weeks thereafter and became undetectable after 9 months, whereas $\mathrm{CD} 4^{+} \mathrm{T}$ cells rose from 120 to 653 cells $/ \mu 1$. In addition, after 1 year of treatment, a GRT performed on HIV-DNA showed the same INI resistance mutations found in 2014. The patient currently maintains undetectable levels of VL.

To the best of our knowledge, this is the first report describing virological success after 1 year of DTG treatment in a patient with archived DTG resistance mutations. Furthermore, this report shows that the concordance between viral compartments may differ profoundly under the selective pressures exerted by antiretroviral drugs. We found that the viral mutants had disappeared from the plasma over time, whereas they persisted in PBMCs after treatment interruption, perhaps due to the different rate of virus turnover in these compartments $[9,10]$. Although the rapid reappearance of DRMs has been hypothesized on treatment reinitiation in the case of archived mutations [8], after more than 1 year from DTG start, our patient is demonstrating excellent virological and immunological responses despite the archived integrase DRM. These findings may be due either to the fact that the HIV-1 genome is often defective in PBMCs after a long-term ART [11], or the current follow-up is too short.

In conclusion, this case confirms that DTG is a potent drug that allows complete viral suppression in a brief time, even in 'difficult patients' with preexisting resistance to INIs. It also suggests that archived DRMs either in plasma and/or in DNA should not limit the use of DTG as a salvage-treatment option. Future studies are needed to better elucidate the mechanism of resistance to DTG, and 
to define guidelines for patients with archived mutations, to minimize the risk of DRM emergence under treatment pressure.

\section{Acknowledgements}

\section{Conflicts of interest}

There are no conflicts of interest.

Laura Mazzuti ${ }^{a}$, Ivano Mezzaroma ${ }^{b}$, Francesca Falasca $^{a}$ and Ombretta Turriziani ${ }^{a}$, ${ }^{a}$ Department of Molecular Medicine, and ${ }^{b}$ Department of Clinical Medicine, Sapienza University, Rome, Italy.

Correspondence to Ombretta Turriziani, PhD, Department of Molecular Medicine, Sapienza University, Viale di Porta Tiburtina 28, 00185 Rome, Italy.

Tel: +3906 49974298;

e-mail: ombretta.turriziani@uniroma1

Received: 20 June 2017; accepted: 23 June 2017.

\section{References}

1. Fantauzzi A, Turriziani O, Mezzaroma I. Potential benefit of dolutegravir once daily: efficacy and safety. HIV AIDS (Auckl) 2013; 5:29-40.

2. Fantauzzi A, Mezzaroma I. Dolutegravir: clinical efficacy and role in HIV therapy. Ther Adv Chronic Dis 2014; 5: 164-177.
3. Wainberg MA, Han YS. HIV-1 resistance to dolutegravir: update and new insights. J Virus Erad 2015; 1:13-16.

4. Eron JJ, Clotet B, Durant J, Katlama C, Kumar P, Lazzarin A, et al., VIKING Study Group. Safety and efficacy of dolutegravir in treatment-experienced subjects with raltegravir-resistant HIV type 1 infection: 24-week results of the VIKING Study. J Infect Dis 2013; 207:740-748.

5. Castagna A, Maggiolo F, Penco G, Wright D, Mills A, Grossberg $\mathrm{R}$, et al. Dolutegravir in antiretroviral-experienced patients with raltegravir- and/or elvitegravir-resistant HIV-1: 24-week results of the phase III VIKING-3 study. I Infect Dis 2014; 210:354-362.

6. Falasca F, Montagna C, Maida P, Bucci M, Fantauzzi A Mezzaroma I, et al. Analysis of intracellular human immunodeficiency virus (HIV)-1 drug resistance mutations in multifailed HIV-1-infected patients treated with a salvage regimen: 72-week follow-up. Clin Microbiol Infect 2013; 19:E318-E321.

7. Noë A, Plum J, Verhofstede C. The latent HIV-1 reservoir in patients undergoing HAART: an archive of pre-HAART drug resistance. / Antimicrob Chemother 2005; 55:410-412.

8. Derache A, Shin HS, Balamane M, White E, Israelski D, Klausner JD, et al. HIV drug resistance mutations in proviral DNA from a community treatment program. PLOS One 2015; 10:e0117430.

9. Turriziani $O$, Andreoni $M$, Antonelli G. Resistant viral variants in cellular reservoirs of human immunodeficiency virus infection. Clin Microbiol Infect 2010; 16:1518-1524.

10. Lee GQ, Swenson LC, Poon AF, Martin JN, Hatano $\mathrm{H}$, Deeks SG, et al. Prolonged and substantial discordance in prevalence of raltegravir-resistant HIV-1 in plasma versus PBMC samples revealed by 454 'deep' sequencing. PLOS One 2012; 7:e46181.

11. Fourati S, Lambert-Niclot S, Soulie C, Malet I, Valantin MA, Descours $B$, et al. HIV-1 genome is often defective in PBMCs and rectal tissues after long-term HAART as a result of APOBEC 3 editing and correlates with the size of reservoirs. J Antimicrob Chemother 2012; 67:2323-2326.

DOI:10.1097/QAD.0000000000001581 